Provided for non-commercial research and education use. Not for reproduction, distribution or commercial use.

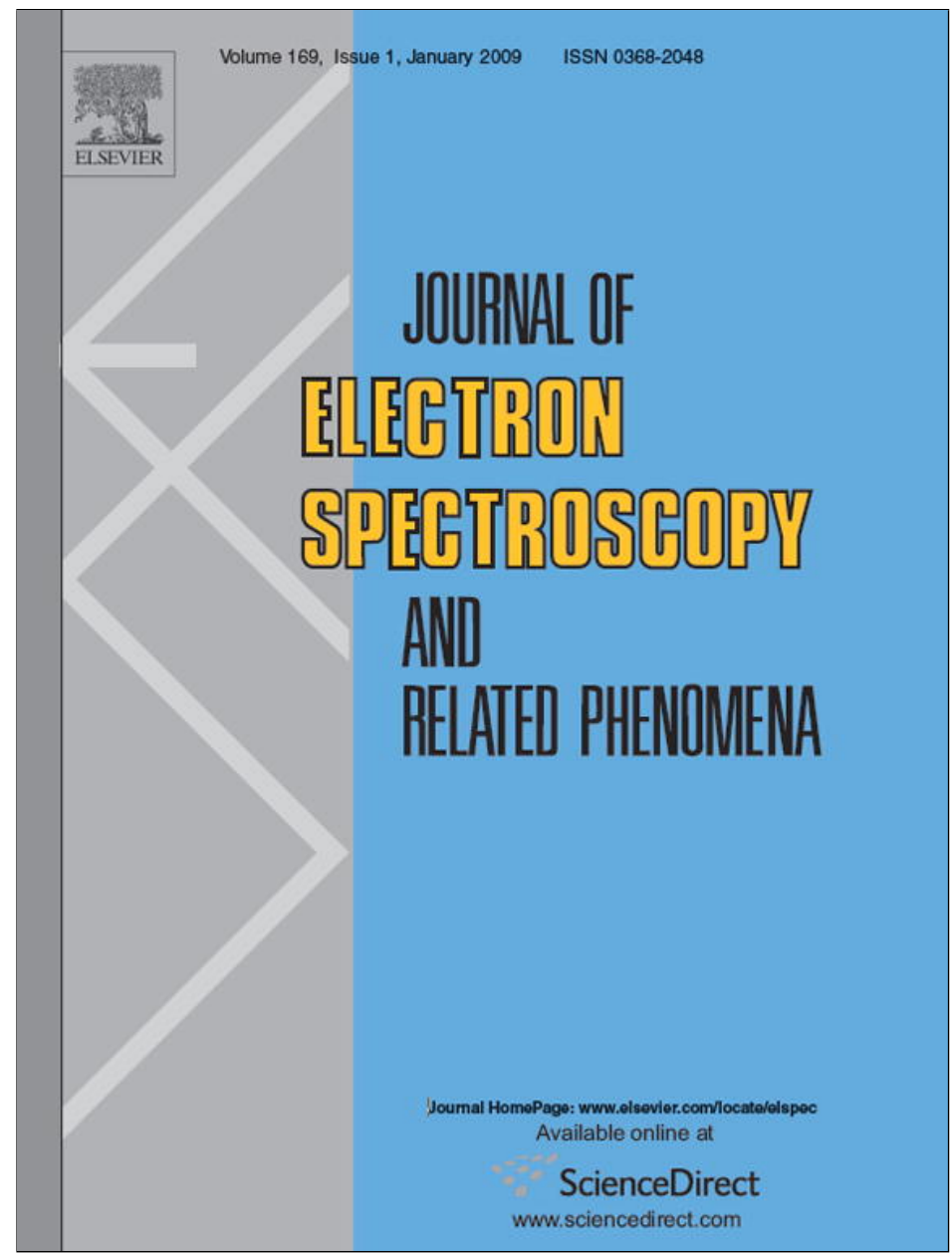

This article appeared in a journal published by Elsevier. The attached copy is furnished to the author for internal non-commercial research and education use, including for instruction at the authors institution and sharing with colleagues.

Other uses, including reproduction and distribution, or selling or licensing copies, or posting to personal, institutional or third party websites are prohibited.

In most cases authors are permitted to post their version of the article (e.g. in Word or Tex form) to their personal website or institutional repository. Authors requiring further information regarding Elsevier's archiving and manuscript policies are encouraged to visit:

http://www.elsevier.com/copyright 


\title{
The role of absorption in large-angle elastic scattering
}

\author{
M.R. Went, M. Vos* \\ Atomic and Molecular Physics Laboratories, Research School of Physical Sciences and Engineering, The Australian National University, Canberra 0200, Australia
}

\section{A R T I C L E I N F O}

\section{Article history:}

Received 15 August 2008

Received in revised form

30 September 2008

Accepted 5 October 2008

Available online 18 October 2008

\section{Keywords:}

Elastic electron scattering

Absorption

Monte Carlo simulations

\begin{abstract}
A B S T R A C T
The measurements of energy loss distributions obtained in electron scattering experiments at high momentum transfer are presented for $\mathrm{Xe}, \mathrm{Ar}$ and methane. The spectra show a large variety of intensity distributions, clearly different from those obtained in measurements at the dipole limit. The fraction of the intensity present in the energy loss distribution compared to the elastic peak is significant, but is in line with the reduction of the elastic cross section due to absorption. It is argued that, if similar effects are present in the condensed phase, they should be dealt with in any quantitative analysis of electron transport in matter, as is often done using Monte Carlo simulations. This argument is worked out in some detail for Reflection Electron Energy Loss Spectroscopy.
\end{abstract}

(c) 2008 Elsevier B.V. All rights reserved.

\section{Introduction}

Simulation of the trajectories of energetic electrons in matter is a subject of considerable importance. It is used to interpret electron spectroscopy and electron microscopy data, electron probe microanalysis, design radiation treatment conditions, etc. For an overview of these simulations in the context of surface science see $[1,2]$. In these simulations the transport problem is generally seen as being governed by two 'independent' processes: elastic and inelastic scattering. The first process changes the propagation direction of the electron due to its interaction with the (screened) electrostatic field of the nucleus. These processes involve no, or very small energy transfers. The second process is due to electronic excitations created by the fast projectile. Its primary effect is a reduction in energy, and for electrons with energies of $1 \mathrm{keV}$ and above the accompanying deflections are small, and these deflections can be neglected relative to those due to elastic scattering. Within this framework one can then subdivide the electron transport problem in two parts: elastic and inelastic scattering. For example, if one studies energetic electrons reflected from a surface, then the differential elastic scattering cross section determines the length of the trajectories below the surface. For a group of trajectories with equal length the inelastic cross section determines which fraction of this group appears without energy loss, and which fraction after $1,2,3, \ldots$ energy loss events.

\footnotetext{
* Corresponding author. Tel: +61 261254985.

E-mail address: maarten.vos@anu.edu.au (M. Vos).
}

In atomic physics it was established quite a while ago, that there is a finite chance that the electron loses energy while it is being deflected by a nucleus. This reduces the intensity of the zero-loss electrons measured at a certain scattering angle, i.e. the differential elastic scattering cross section is reduced. This reduction is referred to as 'absorption'. When measuring the elastic peak these electrons appear 'absorbed' by the atom. In reality very few electron will form a negative ion (in particular if the electron energies are far above the threshold for electronic excitations), so the absorbed electrons will in fact reappear, but at a lower energy.

For low-energy projectiles the momentum transfer, due to inelastic excitations, can be such that the direction of propagation changes significantly. Thus in that case a first-order inelastic process can result in scattering intensity at non-zero energy loss at any angle. For energies of $1 \mathrm{keV}$, and above, the momentum of the projectile is so large, compared to the momentum transfer due to electronic excitations, that it is very unlikely that an electronic excitation causes a deflection over say $45^{\circ}$ or more. Thus intensity of high-energy electrons at large scattering angles and non-zero energy loss (in practise this intensity is measured by comparison to the intensity of the elastic peak at zero energy loss) is always due to higher order processes. In this work we will investigate if we can obtain information about the absorption process by studying the non-zero loss intensity of keV electrons scattering from a gas-phase target over angles of $45^{\circ}$ and more. We will discuss the implications of these measurements for the simulation of electron transport in matter.

The largest possible momentum transfer due to electronelectron collisions is near the 'Bethe ridge'. At $45^{\circ}$ scattering angle the Bethe Ridge corresponds to the projectile losing half its energy. 
None of the experiments reported here are close to the 'Bethe ridge'. For backwards angles the 'Bethe ridge' does not exist as it is kinematically impossible to scatter over more than $90^{\circ}$ from a particle of equal mass. Thus none of the intensity in the spectra reported here is due to simple binary electron-electron collisions.

Elastic scattering is a first order process: the electron interacts with the electrostatic field of the atom (static field approximation), a model that can be refined by including exchange (static exchange approximation). At low and intermediate energies higher order terms become important. For small atoms and at low energies the problem can be solved by considering all possible excitations and solving a set of closed coupling equations [3]. At high energies, and for heavy atoms this is computationally too difficult and the problem is usually attacked using a phenomenological optical potential $[4,5]$. This potential is complex, resulting in a loss of intensity of the elastically scattered electrons, which corresponds to the absorption mentioned above.

Several programs include absorption as an option for calculating differential elastic scattering cross sections of atoms. We use ELSEPA by Salvat et al. [6], and the absorption part of the program is described extensively in ref. [5]. Other approaches exist, e.g. see ref. [7]. The experimental validation of these approaches is usually the comparison with the absolute measurement of the elastic peak intensity as a function of scattering angle as was done for noble gases, see e.g. $[8,9]$. The calculated cross sections with and without absorption are plotted in Fig. 1 for the case of $1 \mathrm{keV}$ electrons scattering from Xe. Clearly the reduction is for large angles of the order of $30 \%$. Of course the cross section is small here, but in many experiments, such as Reflection Electron Energy Loss Spectroscopy (REELS) and Elastic Peak Electron Spectroscopy (EPES) we measure electrons that have scattered over large angles. For quantitative interpretation of these experiments absorption could thus be significant. Absorption is mainly important for large-angle scattering events. In these large $q$ collisions one probes the solid over a distance of the order of $1 / q$ and the scattering intensity originates from near the nucleus. (Classically this corresponds to small impact parameter collisions. Then the electron approaches the nucleus closely, and solid state effects are generally small near a nucleus.) Thus one would expect absorption to be very similar in the gasphase and in condensed matter scattering events. This is in strong contrast to polarisation, a process that has a strong effect on the cross section for small angle (small $q$ or classically a large impact parameter) collisions, and is expected to be very different in the gas and condensed phase.

In these calculations there is no information about the amount of energy that is lost by the scattering electron due to 'absorption'. Here we investigate if we can obtain information about this by simply assuming that the direction of the electron did not change due to the electronic excitation created, and hence the integrated intensity away from the elastic peak divided by the total area is the fractional reduction of the elastic differential cross section due to absorption. This is certainly an approximation, and we will investigate if the results obtained in this way corroborate this assumption. In practice we measure only a finite energy loss window, so the intensity obtained is only a lower limit of the absorbed part of the signal.

\section{Experimental details}

The experimental setup is described in some detail elsewhere [10]. An electron beam with an energy $E_{0}$ between 1 and $6 \mathrm{keV}$ intersects an atomic beam introduced through a needle into the vacuum chamber. An electron gun is positioned at either $45^{\circ}, 90^{\circ}$ or $135^{\circ}$ with respect to the analyzer. The analyzer has a slit lens allowing a range of angles to be decelerated and focussed at the entrance

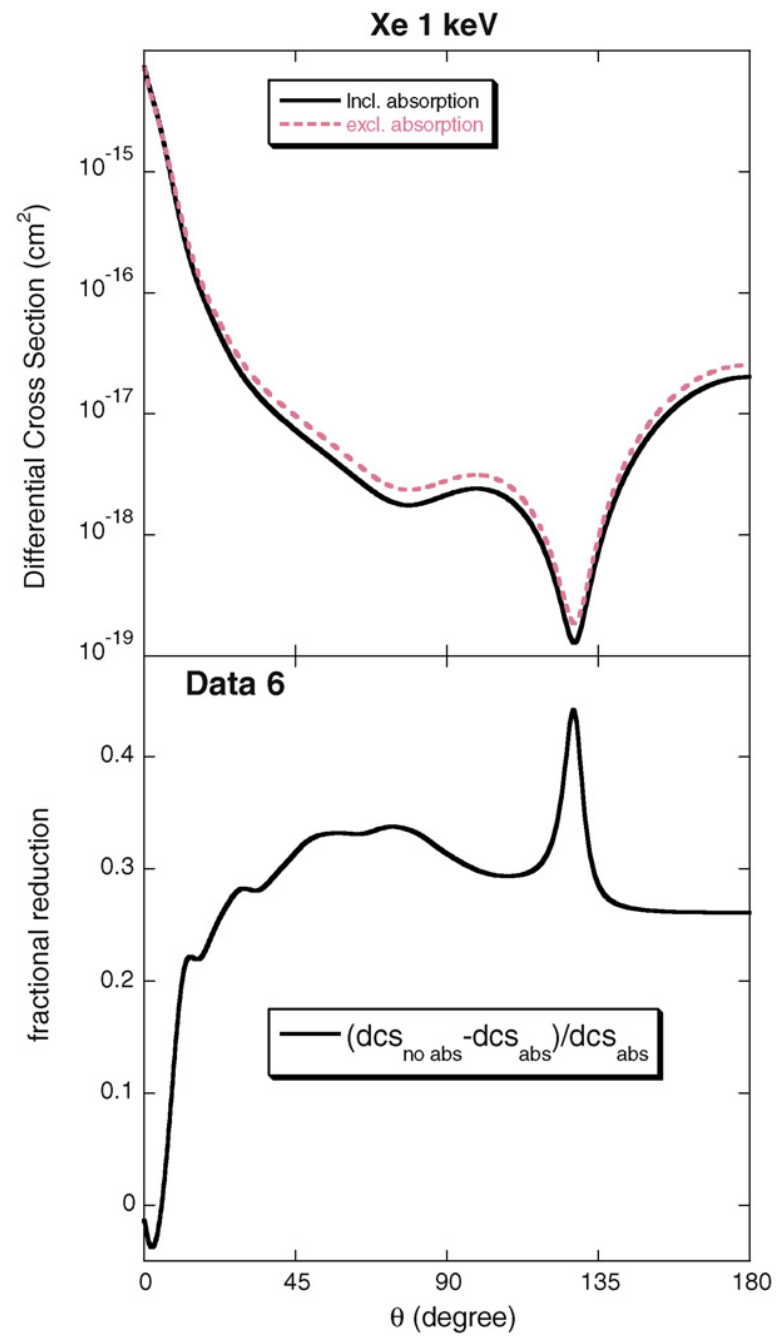

Fig. 1. Calculated cross section with and without absorption for $1 \mathrm{keV} \mathrm{e}^{-}$scattering from Xe (top panel). The lower panel shows the fractional reduction of the differential elastic cross section due to absorption.

plane of a hemispherical analyzer. It operates at a pass energy of $200 \mathrm{eV}$. At the exit plane we have a two-dimensional detector consisting of a Chevron channel plate pair and a resistive anode. This setup allows us to measure an energy window of $\approx 20 \mathrm{eV}$ simultaneously. The potential of the hemisphere is slowly scanned and in this way spectra can be measured over a larger energy range, and the results are compensated for uneven response of the channel plates. The spectrometer was designed to study recoil effects for electron scattering from light atoms (see ref. [10]) but these effects play a negligible role here. For most of the scans a Ta anode was used in the electron gun, with an effective beam current of $1 \mu \mathrm{A}$ resulting in an overall energy resolution of $0.7 \mathrm{eV}$. Some scans used a $\mathrm{BaO}$ anode, operating at a lower temperature and in this way we can obtain energy resolution of $0.5 \mathrm{eV}$, using beam currents around $250 \mathrm{nA}$. Each measurement consisted of a signal run and a background run. During the signal run the gas was directed through the needle, ending just below the interaction region. During the background run the gas entered the chamber far away from the interaction region. The result presented here are the difference between signal and background run. Counts in the background run are mostly due to the halo of the electron beam hitting the needle, and interaction with the background gas. Data collection for a given $E_{0}$ and scattering angle typically takes several days. 


\section{Results}

\subsection{Experimental data}

Spectra obtained for Xe at different angles and energies are shown in Fig. 2. Not all measurements were done over the same scan range. Measurement times were different, and the number of true counts in the elastic peak ranges between $10^{6}$ and $10^{7}$. For easy comparison all elastic peaks were normalised to $10^{6}$ counts, and the zero of the energy scale was taken at the maximum of the elastic peak. For a resolution of $0.7 \mathrm{eV}$ and a step size of $0.1 \mathrm{eV}$ this means that the top of the elastic peak is near $1.1 \times 10^{5}$ counts. The inelastic signal is, at any given energy, roughly 1000 times smaller than the maximum elastic signal strength. For this reason it is essential to have two-dimensional detection in the analyzer, in order to limit the data-acquisition time for each measurement to a few days or so. In the following we give a qualitative description of the obtained data. There exists however, extensive literature of atomic physics studies of electron scattering at or near the dipole limit (zero momentum transfer), often employing much better energy resolution as under these conditions the cross sections are much more favourable. We will refer to examples of this literature to indicate which aspects are the same at low and at high momentum transfer, but we do not pretend to give a complete review of the literature of electron scattering at or near the dipole limit.

The spectra can be divided into different regions according to which shell contributes most to the loss features. It turns out that the relative intensity of the different regions varies substantially with the scattering conditions. The outermost electrons of Xe have a binding energy of $12.1 \mathrm{eV}\left(5 \mathrm{p}_{3 / 2}\right)$ and $13.4 \mathrm{eV}\left(5 \mathrm{p}_{3 / 2}\right)$. The first feature in the loss spectrum is due to creation of a discrete state (Rydberg state) near $8.5 \mathrm{eV}$ energy loss, followed by at least two more discrete states. Excitation to the continuum ('ionisation') of the $5 p_{3 / 2}$ electrons becomes possible above $12.1 \mathrm{eV}$ energy loss. At lower momentum transfer these spectra have been measured before at much higher energy resolution and then many more states are resolved, see e.g. [11].

The next discrete state is near $20.5 \mathrm{eV}$, and will be due to excitation of a $5 \mathrm{~s}$ electron (binding energy $23.3 \mathrm{eV}$ ). In the dipole limit these 5s-related structures are not visible [12]. The intensity increase in the loss spectrum around these energies is particularly strong at the smallest angle of $45^{\circ}$ studied here, and at lower energies. Then, although the momentum transfer and scattering angle are small (in the context of this paper) the dipole forbidden transitions are seen most clearly. This is an intriguing fact.

With increasing energy loss the intensity of the loss spectrum decreases until new discrete states are observed at 66.3 and $68.3 \mathrm{eV}$. These discrete states are associated with the excitation of the $4 d_{5 / 2}$ and $4 \mathrm{~d}_{3 / 2}$ electrons (binding energy 67.5 and $69.5 \mathrm{eV}$ ). These discrete states are followed by a very broad peak due to excitation of these $4 \mathrm{~d}$ electrons to the continuum, which is enhanced due to the presence of unoccupied $4 \mathrm{f}$ levels. Both the discrete states and broad peak are known from lower momentum transfer measurements [13]. Near $145 \mathrm{eV}$ the $4 \mathrm{p}$ electrons could come into play, but we have not seen any clear evidence of discrete states associated with these electrons, but the significant intensity above $140 \mathrm{eV}$ makes it likely that these electrons contribute as well.

In principle there are two ways that intensity at non-zero energy loss can be produced. The electronic excitation can occur in the atom that scatters the electron elastically, or the excitation can happen due to inelastic scattering at a different atom, before or after the elastic deflection. Fortunately it is easy to distinguish between these two possibilities. Increasing the gas flow through the needle (as can be monitored by observing the pressure in

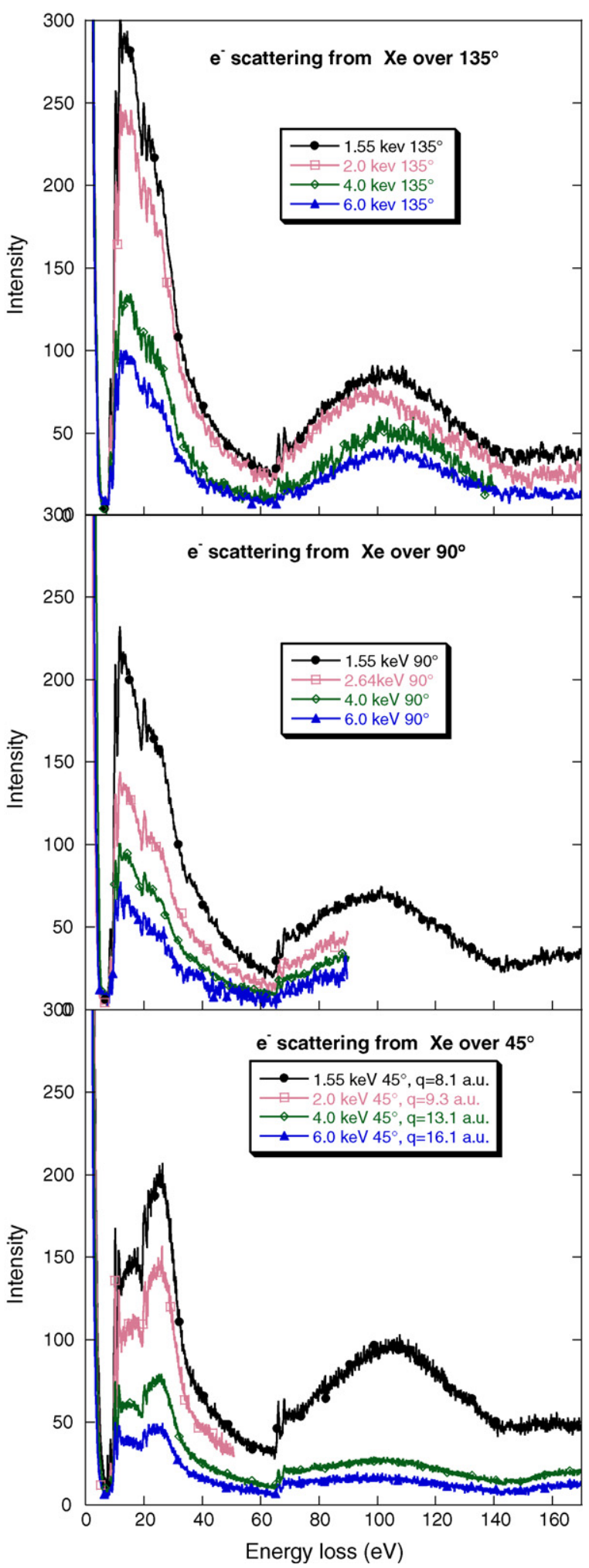

Fig. 2. The spectra obtained at three different scattering angles, for the energies as indicated. The elastic peak was normalised, for convenience, to $10^{6}$ counts in all cases. A Ta emitter was used in these cases and the energy resolution was approximately $0.7 \mathrm{eV}$. 


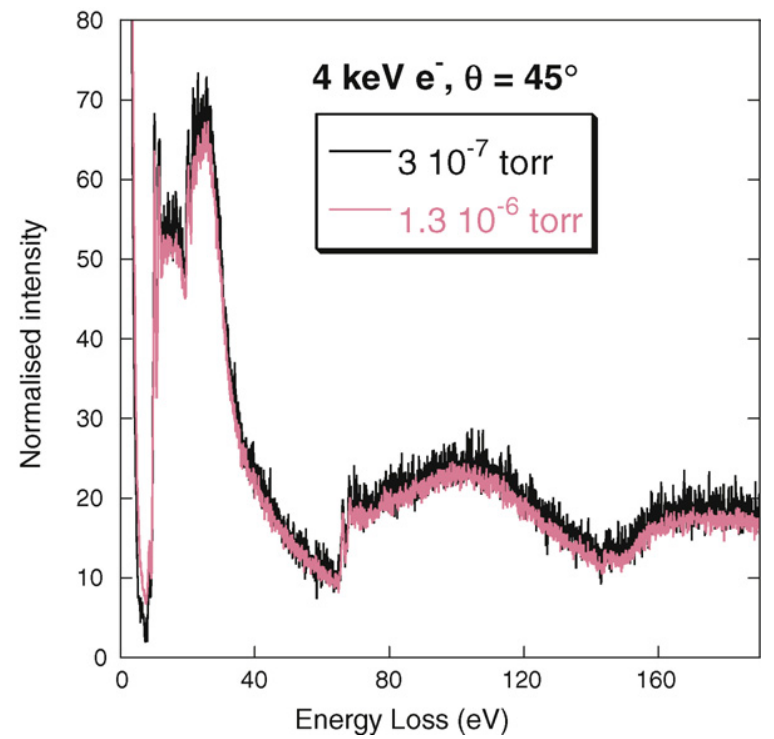

Fig. 3. The intensity of the loss feature at two different operating pressures. The elastic peak area is in both cases normalised to $10^{6}$ counts. $\left(E_{0}=4 \mathrm{keV}, \theta=45^{\circ}\right)$

the chamber) will increase the density of atoms in the interaction region and hence increase the probability that an elastically deflected electron interacts inelastically with another Xe atom. The results in Fig. 3 show that the intensity of the loss feature (and its shape) is independent from the Xe flow rate. Moreover for processes involving two atoms it would be very hard to explain why the shape of the loss distribution depends so strongly on the scattering angle.

These findings are not restricted for Xe. We repeated the experiment with $\mathrm{Ar}$ and $\mathrm{CH}_{4}$, and these results are shown in Fig. 4. For $\mathrm{Ar}$ the low loss region resembles that of Xe, although the exact peak positions are different. Again the intensity due to the outermost level (3p) increases slower with decreasing $E_{0}$ than the intensity due to adjacent $3 \mathrm{~s}$ electrons. The outermost core levels for Ar are the $2 \mathrm{p}$ levels near $250 \mathrm{eV}$. Here we see a sudden increase of intensity, with signs of 2 discrete levels with minor intensity. For Xe we did not see a such a clear fingerprint of contributions due to its $4 p$ electrons (binding energy $\approx 146 \mathrm{eV}$ ) but a similar step as due to the Ar2p electrons is probably hidden by the decline of the peak related to the excitations of $4 \mathrm{~d}$ electrons, which is centred around $105 \mathrm{eV}$.

Methane is a molecule with a closed shell configuration. Spectra obtain for methane resemble the energy loss structures for Xe and Ar. There are two onsets, at slightly lower energy than the 2 valence band orbitals ( $1 t_{2}$ at $14 \mathrm{eV}$ and $2 A_{1}$ at $23 \mathrm{eV}$ ). Again in energy loss measurements near the dipole limit the second onset, related to an orbital with s symmetry, is not evident [14]. The carbon $\mathrm{K}$ region was studied in a separate run. Its results are shown as an inset in Fig. 4. Two strong peaks are observed. The first one, near $287 \mathrm{eV}$ is seen in high-resolution dipole work as a very weak feature [15] and is associated with the dipole forbidden $1 \mathrm{~s} \rightarrow 3 \mathrm{~s}$ transition. Again it is a relatively strong feature in the current study. The second strong feature, near $288 \mathrm{eV}$, corresponds to the $1 \mathrm{~s} \rightarrow 3 \mathrm{p}$ transition and is also a dominant feature at low momentum transfer [15].

Some of the measurements were repeated using a $\mathrm{BaO}$ cathode. Due to its lower operating temperature the electron beam produced by a $\mathrm{BaO}$ cathode has a smaller thermal spread. Hence the resolution of the experiment increased from 0.7 to $0.5 \mathrm{eV}$ FWHM, as obtained from the width of the elastic peak. Results for the low loss region are shown in Fig. 5. It shows the discrete states mentioned in the text more clearly. Notice also the dip in the spectrum before the discrete state related to the s levels. These discrete
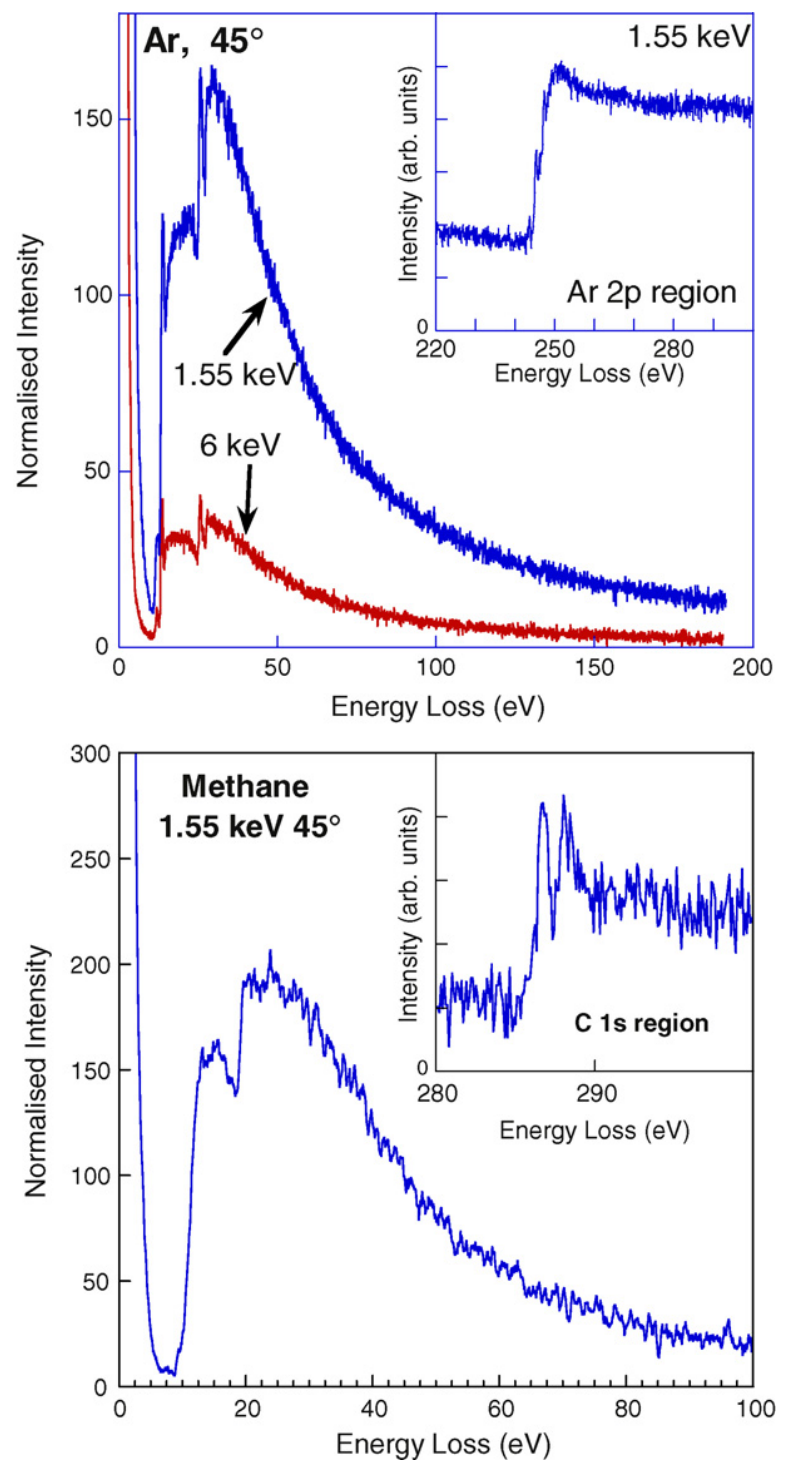

Fig. 4. Spectra for electrons scattered over $45^{\circ}$ from $\mathrm{Ar}$ and methane

states are auto-ionising as they are degenerate with p electrons excited to the continuum. Interference between these channels causes the Fano line-shapes [16] with the characteristic dip before the peak. Using a small scattering angle $\left(3^{\circ}\right)$ and lower energies $(30-500 \mathrm{eV})$ these states were investigated at high resolution by Yuan et al. [17]. Their spectra showed large variations in intensities and shapes, and extrapolating to our measurement conditions is non-trivial.

\subsection{Intensity of loss spectra}

Now we want to compare the area in the zero loss peak $I_{0}$ with the total area at non-zero energy loss features $I_{\text {loss }}$. Ideally the measurement would extend over all kinetic energies, but with our spectrometer we are limited to a range of $200 \mathrm{eV}$ or so. In Fig. 6 we plot the fraction of the intensity that is in the loss area integrated from 8 to $190 \mathrm{eV}$ for Xe. Clearly this fraction reduces with increasing energy, but it seems to be fairly independent of the scattering angle.

We assume that the intensity at non-zero energy loss is a consequence of absorption. In a somewhat naive model we can then compare the normalised difference between the calculated elastic 

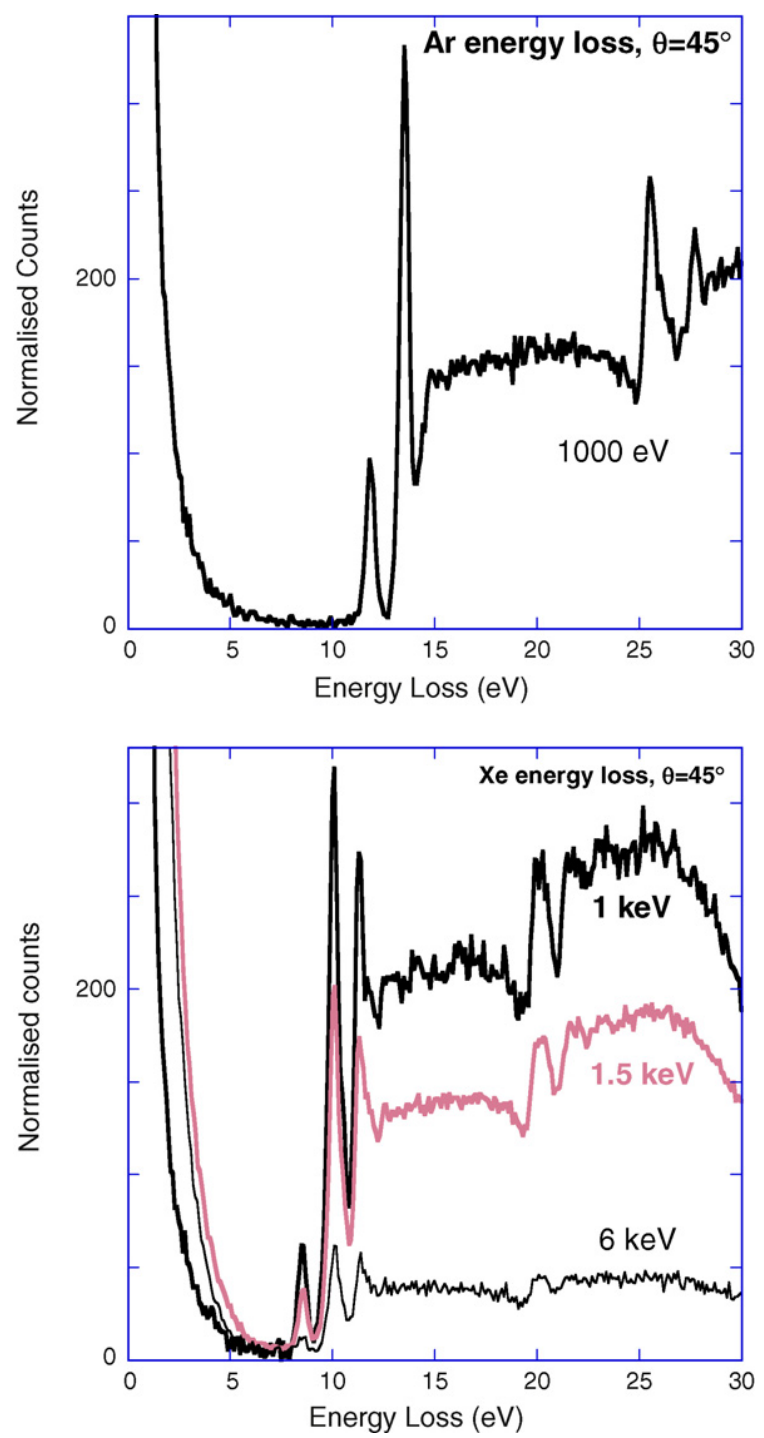

Fig. 5. Low energy loss region of $\mathrm{Ar}$ and $\mathrm{Xe}$ as measured with a $\mathrm{BaO}$ cathode, at energies as indicated. All spectra are normalised such that the elastic peak area is $10^{6}$.

cross section with and without absorption with these data. Thus

$\frac{(\mathrm{d} \sigma / \mathrm{d} \omega)_{\text {noabs }}-(\mathrm{d} \sigma / \mathrm{d} \omega)_{a b s}}{(\mathrm{~d} \sigma / \mathrm{d} \omega)_{a b s}}=\frac{I_{\text {loss }}}{I_{0}}$

These values, obtained from ELSEPA are also plotted in Fig. 6. The calculated difference depends in a similar way on $E_{0}$ as $I_{\text {loss }} / I_{0}$. Also the calculated ratio seems to be fairly independent of the scattering angle. The measured values need to be multiplied by a factor between 2 and 2.5 to fall on the calculated ratio. This is due, at least in part, to the fact that we measured only over a finite energy loss range. Unfortunately the spectrometer is not built for measurements over all energies.

There are some complications that put doubt on the simple interpretation sketched above. For example, the total elastic cross section without absorption, $\sigma_{\text {no abs }}^{e l}$ is not equal to the total elastic cross section including absorption $\sigma_{a b s}^{e l}$ plus the absorption cross section $\sigma^{a b s}$, as calculated by ELSEPA. However, the fairly constant fractional reduction of the cross section (see Fig. 1) for large scattering angles corresponds fairly well to the ratio of $\sigma^{a b s}$ and $\sigma_{a b s}^{e l}$. Another possible complication is that absorption processes may

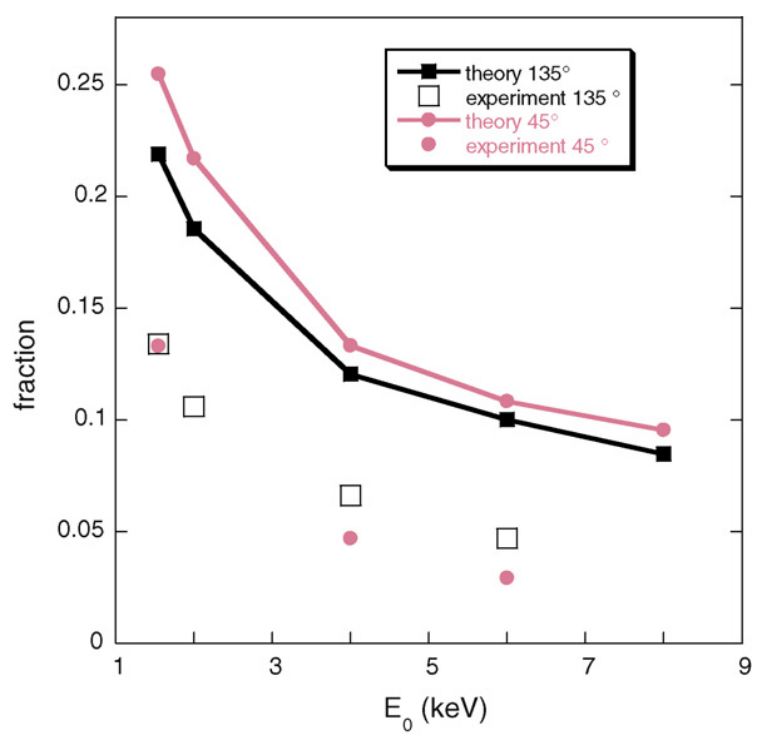

Fig. 6. A comparison for Xe of the fraction of the intensity at non-zero energy loss (integrated from 8 to $190 \mathrm{eV}$ ) and in the elastic peak with the fractional reduction of the elastic cross section due to absorption.

not just shift intensity away from zero energy loss, but may also influence the angular distribution, even at these high energies.

\section{Implications for Monte Carlo simulations}

In the previous section we outlined the case that information about absorption on elastic scattering can be obtained by studying large-angle scattering events in the gas-phase. These measurements provide information about the microscopic picture required to describe absorption fully quantitatively, not just how many electrons lose energy while scattering elastically, but also how much energy is lost and which shells contribute to absorption.

Here we want to focus briefly on what this means for electron transport simulations in solids. We assume, as these collision conditions involve large momentum transfer, that these collisions are very localised to the nuclear region, and hence that elastic deflections in condensed matter are similarly affected by absorption. In the literature there has been a discussion on whether the differential elastic scattering cross section that should be used in solids should include absorption or not (e.g. [2]). The answer will depend in part on which aspects are important (e.g. only elastic peak is measured as in EPES, only small angle scattering is important as in transmission electron energy loss spectroscopy). In some cases inclusion of absorption will entail more than just a change in the differential elastic cross section. To be concrete we now focus in some more detail on the case of REELS.

In REELS one often separates the elastic and inelastic part of the problem. If one is interested in the energy loss spectrum over a range that is small compared to $E_{0}$, then one can assume that the differential elastic scattering cross section is constant over the energy range of interest. One simulates, using Monte Carlo techniques, the length of the trajectories using elastic scattering cross sections, and subsequently calculates, based on the mean free path the probability that the electron made $0,1,2, \ldots n$ inelastic excitations (in addition to possible surface excitations, which we neglect here to keep the discussion simple). In these experiments all electrons detected have been scattered at least once over a significant angle. For these angles, as we have seen, the probability of energy loss due to absorption is significant $(\simeq 30 \%$ at $1 \mathrm{keV}$ for scattering from heavy atoms). Hence absorption could be an additional cause of the intensity at non-zero energy loss. 
Without absorption the Monte Carlo procedure is straight forward. We have $\lambda_{e l}=1 / n \sigma_{e l}$ with $n$ the atomic density and $\sigma_{e l}$ the total elastic cross section. Based on $\lambda_{e l}$ we choose the path length to the next deflection. Then based on the differential cross section $\mathrm{d} \sigma / \mathrm{d} \Omega(\theta)$ we use a Monte Carlo method to choose the next deflection. This procedure is repeated until an end condition has been reached.

If one wants to include absorption in a Monte Carlo procedure, we have to be a bit more careful, and we suggest the following procedure: Let $\sigma_{e l}^{i n}$ and $\sigma_{e l}^{e x}$ be the elastic cross section including and excluding absorption. The mean distance between deflections by nuclei (either with or without energy loss) is then $\lambda_{e l}=1 /\left(n \sigma_{e l}^{e x}\right)$. Subsequently one uses Monte Carlo method to select a scattering angle based on the shape of $\mathrm{d} \sigma_{e l}^{e x} / \mathrm{d} \Omega$. Then from the ratio of $\mathrm{d} \sigma_{e l}^{e x} / \mathrm{d} \Omega$ and $\mathrm{d} \sigma_{e l}^{i n} / \mathrm{d} \Omega$ at that angle $\theta$ one uses Monte Carlo procedure to select if or if not energy was lost due to the deflection. For example, if absorption reduces the cross section by $30 \%$ then in 3 out of 10 cases energy will be lost during the deflection. If energy is lost then one uses Monte Carlo technique to choose a value of the lost energy from an appropriate distribution. Subsequently one proceeds with the next deflection, summing all energy loss events due to elastic deflections for a given trajectory. This energy loss is in addition of the energy loss due to the inelastic mean free path, which still has to be calculated in the conventional way. The energy loss due to absorption distinguishes itself from other inelastic losses in that it is strongly correlated with large-angle elastic deflections. It is thus not possible to describe these loss effects satisfactorily by just modifying the inelastic mean free path and/or the loss function.

The unresolved problem in the procedure sketched above is the 'appropriate energy loss distribution'. As we have seen for Xe its shape depends on the deflection angle, and currently there is no theoretical recipe for obtaining this distribution. For most elements one can not easily determine these shapes experimentally. As these losses are much more localised than the inelastic energy loss processes in a solid, we have no reason to assume that the inelastic loss function is a good estimate for the absorption losses. Indeed for $\mathrm{Ar}$ and Xe the losses due to absorption extend well beyond $100 \mathrm{eV}$ where, for example, the 'universal loss function' proposed by Tougaard [18] to describe inelastic loss distribution becomes very small at these energies.

Another indication that the picture sketched here is too simple is the fact that at forward angles the elastic differential cross section with absorption can be slightly larger than the one without absorption (see Fig. 1). A satisfactorily description of this phenomenon requires a more formal quantum-mechanical approach.

\section{Conclusion}

This paper is an exploratory study of the intensity distribution at non-zero energy loss that can be observed in high-momentumtransfer scattering experiments. The fractional changes in this intensity with $E_{0}$ resembles the dependence of 'absorption' on energy. As only a limited energy loss range is studied we can not say if the fraction of the intensity at non-zero energy loss is equal to the fractional reduction of the DCS due to absorption. Decrease of intensity with increasing energy loss is slow, hence it is conceivable that the measured intensity for a measurement extending over all energies would approach the reduction in the elastic cross section due to absorption as shown in Fig. 6. We would not like to suggest different values of $A_{a b s}$ the empirical constant that determines the absorption strength in ELSEPA.

To our knowledge very little theoretical work exists on the energy loss of electrons scattering at high-momentum transfer. A different approach can be found in the work of Hidalgo and Geltman [19] on the intensity at large scattering angle for electrons scattering from helium. They treated the outgoing electrons as a Coulomb wave, and include the influence of the nucleus in this way. The probability of exciting discrete final states is then calculated in the first Born approximation. This leads to much more intensity in these excited states at large scattering angle, compared to calculations using plane waves for the outgoing electron.

The measured spectra display a myriad of shapes, and if it becomes possible to develop a quantitative theory for these scattering experiments, then measurements, as described here, will be of great value to test these calculations. For now our description has been rather qualitative, focussing mainly on the differences in shape as seen here, and in the dipole limit.

We believe however, that with increasing sophistication of Monte Carlo simulations for electron transport in solids, and more detailed comparison between experiment and simulations, it is timely to start investigating if absorption effects have to be taken into account, if comparison between simulation and theory approaches a quantitative level, as was the case in recent REELS studies (see e.g. [20]), especially for studies dealing with high$Z$ materials. This paper gives some experimental input to open a discussion addressing this problem.

\section{Acknowledgments}

The authors want to thank Erich Weigold, Bob McEachran and Jerry Seidler for stimulating discussions and carefully reading the manuscript. This research was made possible by a grant of the Australian Research Council.

\section{References}

[1] W.S.M. Werner, Surf. Interface Anal. 31 (2001) 141-176.

[2] A. Jablonski, Prog. Surf. Sci. 79 (2005) 3.

[3] I. Bray, D.H. Madison, I.E. McCarthy, Phys. Rev. A 41 (1990) 5916

[4] J.B. Furness, I.E. McCarthy, J. Phys. B: At. Mol. Phys. 6 (1973) 2280-2291.

[5] F. Salvat, Phys. Rev. A 68 (2003) 12708.

[6] F. Salvat, A. Jablonski, C.J. Powell, Comput. Phys. Commun. 165 (2005) 157190.

[7] F. Blanco, G. García, Phys. Rev. A 67 (2003) 022701

[8] R.H.J. Jansen, F.J. de Heer, H.J. Luyken, B. van Wingerden, H.J. Blaauw, J. Phys. B: At. Mol. Phys. 9 (1976) 185-212.

[9] R.H.J. Jansen, F.J. de Heer, J. Phys. B: At. Mol. Phys. 9 (1976) 213-226.

[10] M. Vos, M. Went, G. Cooper, C. Chatzidimitriou-Dreismann, J. Phys. B 41 (2008) 135204.

[11] S. Urpelainen, M. Huttula, P. Kovala, A. Makinen, A. Calo, S. Aksela, H. Aksela, J. Electron Spectrosc. Relat. Phenom. 156-158 (2007) 145-149.

[12] W.F. Chan, G. Cooper, X. Guo, G.R. Burton, C.E. Brion, Phys. Rev. A 46 (1992) 149-171.

[13] H.M. Boechat-Roberty, J.D. Freitas, D.P. Almeida, G.G.B. de Souza, J. Phys. B: At Mol. Opt. Phys. 35 (2002) 1409-1420.

[14] J.W. Au, G. Cooper, G.R. Burton, T.N. Olney, C.E. Brion, Chem. Phys. 173 (1993) 209-239.

[15] M. Tronc, G.C. King, F.H. Read, J. Phys. B: At. Mol. Phys. 12 (1979) 137-157.

[16] U. Fano, J.W. Cooper, Rev. Mod. Phys. 40 (3) (1968) 441-507.

[17] Z.-S. Yuan, Y. Sakai, N. Umeda, Y. Fujita, T. Takayanagi, C. Yamada, N. Nakamura, S. Ohtani, L.-F. Zhu, K.-Z. Xu, Mol. Opt. Phys. 39 (2006) 5097-5103.

[18] S. Tougaard, Solid State Commun. 61 (1987) 547-549.

[19] M.B. Hidalgo, S. Geltman, J. Phys. B: At. Mol. Phys. 5 (1972) 617-626.

[20] W. Werner, M.R. Went, M. Vos, Surf. Sci. 601 (2007) L109-113. 